\title{
A Subclass of Analytic Functions Related to $k$-Uniformly Convex and Starlike Functions
}

\author{
Saqib Hussain, ${ }^{1}$ Akhter Rasheed, ${ }^{2}$ Muhammad Asad Zaighum, ${ }^{2}$ and Maslina Darus ${ }^{3}$ \\ ${ }^{1}$ Department of Mathematics, COMSATS Institute of Information Technology, Abbottabad, Pakistan \\ ${ }^{2}$ Department of Mathematics \& Statistics, Riphah International University, Islamabad, Pakistan \\ ${ }^{3}$ School of Mathematical Sciences, Faculty of Science and Technology, Universiti Kebangsaan Malaysia, \\ 43600 Bangi, Selangor, Malaysia
}

Correspondence should be addressed to Saqib Hussain; saqib_math@yahoo.com

Received 26 January 2017; Accepted 20 April 2017; Published 23 May 2017

Academic Editor: Maria Alessandra Ragusa

Copyright (C) 2017 Saqib Hussain et al. This is an open access article distributed under the Creative Commons Attribution License, which permits unrestricted use, distribution, and reproduction in any medium, provided the original work is properly cited.

We investigate some subclasses of $k$-uniformly convex and $k$-uniformly starlike functions in open unit disc, which is generalization of class of convex and starlike functions. Some coefficient inequalities, a distortion theorem, the radii of close-to-convexity, and starlikeness and convexity for these classes of functions are studied. The behavior of these classes under a certain modified convolution operator is also discussed.

\section{Introduction}

Let $\mathscr{A}$ be the class of all analytic functions $f$ in open unit disc $\Delta=\{z:|z|<1\}$, normalized by $f(0)=0$ and $f^{\prime}(0)=1$. Thus, any $f \in \mathscr{A}$ has the following Maclaurin's series:

$$
f(z)=z+\sum_{n=2}^{\infty} a_{n} z^{n}
$$

A function $f$ is said to be univalent if it never takes same value twice. By $\delta$ we mean the subclass of $\mathscr{A}$ which is composed of univalent functions. By $\mathcal{S} \mathscr{T}$ and $\mathscr{C} \mathscr{V}$ we mean the well-known subclasses of $\mathscr{A}$ that are, respectively, starlike and convex.

In 1991, Goodman [1, 2] introduced the classes $\mathscr{U} \mathscr{C} \mathscr{V}$ and $\mathscr{U} \mathcal{S} \mathscr{T}$ of uniformly convex and uniformly starlike functions, respectively. A function $f \in S$ is uniformly convex if $f(z)$ maps every circular arc $\gamma$ contained in $\Delta$ with center $\zeta \in \Delta$ onto a convex arc. The function $f \in S$ is uniformly starlike if $f(z)$ maps every circular arc $\gamma$ contained in $\Delta$ with center $\zeta \in \Delta$ onto a starlike arc with respect to $f(\zeta)$. A more useful representation of $\mathscr{U} \mathscr{C} \mathscr{V}$ and $\mathscr{U} \mathcal{S} \mathscr{T}$ was given in [3-6] as

$$
\begin{aligned}
f & \in \mathscr{U} \mathscr{C} \mathscr{V} \Longleftrightarrow f \in \mathscr{A}, \\
\operatorname{Re}\left(\frac{\left(z f^{\prime}(z)\right)^{\prime}}{f^{\prime}(z)}\right)>\left|\frac{z f^{\prime \prime}(z)}{f^{\prime}(z)}\right|, &
\end{aligned}
$$

$z \in \Delta$.

$$
\begin{aligned}
f & \in \mathcal{U S T} \mathscr{T} \Longleftrightarrow f \in \mathscr{A}, \\
\operatorname{Re}\left(\frac{z f^{\prime}(z)}{f(z)}\right)>\left|\frac{z f^{\prime}(z)}{f(z)}-1\right|, &
\end{aligned}
$$$$
z \in \Delta \text {. }
$$

In 1999, for $k \geq 0$, Kanas and Wisniowska [7] introduced the class $k-\mathscr{U} \mathscr{C} \mathscr{V}$ and $k-\mathscr{U} \mathcal{S} \mathscr{T}$ as

$$
\begin{aligned}
f \in k-\mathscr{U} \mathscr{C} \mathscr{V} & \Longleftrightarrow \\
z f^{\prime} \in k-\mathscr{U} \mathcal{S} \mathscr{T} & \Longleftrightarrow f \in \mathscr{A}, \\
\operatorname{Re}\left(\frac{\left(z f^{\prime}(z)\right)^{\prime}}{f^{\prime}(z)}\right)>k\left|\frac{z f^{\prime \prime}(z)}{f^{\prime}(z)}\right|, &
\end{aligned}
$$

$$
z \in \Delta \text {. }
$$

Observe that $0-\mathscr{U} \mathscr{C} \mathscr{V} \equiv \mathscr{C} \mathscr{V}, 0-\mathscr{U} \mathcal{S} \mathscr{T} \equiv \mathscr{U} \mathcal{S} \mathscr{T}$ and $1-\mathcal{U} \mathscr{C} \mathscr{V} \equiv \mathscr{U} \mathscr{C} \mathscr{V}, 1-\mathscr{U} \mathcal{S} \mathscr{T} \equiv \mathscr{U} \mathcal{S} \mathscr{T}$.

For fixed $k \geq 0$, these classes have a nice geometrical representation; for detail see [7-9]. 
A lot of authors obtain very useful properties of $\mathscr{U} \mathscr{C} \mathscr{V}$ and $\mathscr{U} \mathscr{S} \mathscr{T}$ and their generalization in several direction; for example, see $[1,2,7,8,10,11]$ and reference cited therein.

For $(0 \leq \alpha<1)$, in [4] (see also [12]), Ronning introduced the following two important subclasses $k-\mathscr{U} \mathscr{S} \mathscr{T}(\alpha)$ and $k-$ $\mathscr{U} \mathscr{C} \mathscr{V}(\alpha)$ as

$$
\begin{gathered}
f \in k-\mathcal{U S T}(\alpha) \Longleftrightarrow \\
\operatorname{Re}\left\{\frac{z f^{\prime}(z)}{f(z)}-\alpha\right\}>k\left|\frac{z f^{\prime}(z)}{f(z)}-1\right|, \\
f \in k-\mathcal{U} \mathscr{C} \mathscr{V}(\alpha) \Longleftrightarrow \\
z f^{\prime} \in k-\mathcal{U S T}(\alpha) .
\end{gathered}
$$

Recently in [13] El-Ashwah et al. introduced two important subclass $k-\mathscr{U} \mathscr{C} \mathscr{V}(\alpha, \beta)$ and $k-\mathscr{U} \delta \mathscr{T}(\alpha, \beta)$ of $k$ uniformly convex starlike functions as

$$
\begin{gathered}
f \in k-\mathscr{U} \mathscr{C V}(\alpha, \beta) \Longleftrightarrow \\
\operatorname{Re}\left\{\frac{\left(z f^{\prime}(z)\right)^{\prime}}{f^{\prime}(z)}-\alpha\right\}>k\left|\frac{\left(z f^{\prime}(z)\right)^{\prime}}{f^{\prime}(z)}-\beta\right|, \\
f \in k-\mathscr{U} \mathcal{S} \mathscr{T}(\alpha, \beta) \Longleftrightarrow \\
z \in \Delta, \\
\operatorname{Re}\left\{\frac{z f^{\prime}(z)}{f(z)}-\alpha\right\}>k\left|\frac{z f^{\prime}(z)}{f(z)}-\beta\right|,
\end{gathered}
$$$$
z \in \Delta \text {, }
$$

where $(0 \leq \alpha<\beta \leq 1)$ and $k(1-\beta)<1-\alpha$.

Let $f_{j}(j=1,2, \ldots)$ be defined by

$$
f_{j}(z)=z+\sum_{n=2}^{\infty} a_{n, j} z^{n}, \quad a_{n, j} \geq 0 ; j \in N ;
$$

then the modified Hadmard product of $f_{1}(z)$ and $f_{2}(z)$ is defined by

$$
\left(f_{1} * f_{2}\right)(z)=z-\sum_{n=2}^{\infty} a_{n, 1} a_{n, 2} z^{n}
$$

We denote $\mathscr{T}$ by subclass of $\mathcal{S}$ consisting of functions having all negative coefficients in their Maclaurin's series expansions, so any $f \in \mathscr{T}$ has a series of the form:

$$
f(z)=z-\sum_{n=2}^{\infty}\left|a_{n}\right| z^{n}, \quad z \in \Delta .
$$

Let $\mathscr{V}_{\eta}$ be the class of functions $f \in \mathcal{S}$ given in (1) for which $\arg \left(a_{n}\right)=\pi+(n-1) \eta, n \geq 2$. Note that $\mathscr{V}_{0}=\mathscr{T}$ [11].

In recent years, more and more researchers are interested in the above defined classes (see $[9,11,14-22]$ ).

In this paper, by taking inspiration from the above cited paper, we introduce some new subclasses of analytic functions and obtain some interesting results.
Definition 1. For $(0 \leq \alpha<\beta \leq 1), 0 \leq \delta<1, k(1-\beta)<1-\alpha$, and $0 \leq \lambda<1$, a function $f \in \mathcal{S}$ is in class $k-\mathcal{U}(\alpha, \beta, \lambda, \delta)$ if and only if

$$
\begin{aligned}
& \operatorname{Re}\left\{\frac{(1-\delta) z f^{\prime}+\delta\left(z f^{\prime}+(1+2 \lambda) z^{2} f^{\prime \prime}+\lambda z^{3} f^{\prime \prime \prime}\right)}{(1-\delta) f+\delta\left(z f^{\prime}+\lambda z^{2} f^{\prime \prime}\right)}\right. \\
& -\alpha\} \\
& \quad \geq k \mid \frac{(1-\delta) z f^{\prime}+\delta\left(z f^{\prime}+(1+2 \lambda) z^{2} f^{\prime \prime}+\lambda z^{3} f^{\prime \prime \prime}\right)}{(1-\delta) f+\delta\left(z f^{\prime}+\lambda z^{2} f^{\prime \prime}\right)} \\
& -\beta \mid, \quad z \in \Delta .
\end{aligned}
$$

Also

$$
k-\mathscr{V} \mathscr{U}_{\eta}(\alpha, \beta, \lambda, \delta)=k-\mathscr{U}(\alpha, \beta, \lambda, \delta) \cap \mathscr{V}_{\eta} .
$$

It is worth mentioning that, for special values of parameters, these classes were extensively studied by many authors; here we mention few of them.

(1) $k-\mathscr{U}(\alpha, \beta, \lambda, 1)=k-U(\lambda, \beta, \alpha)[21]$.

(2) $k-\mathscr{V} \mathscr{U}_{0}(\alpha, \beta, \lambda, 1)=k-V U_{\eta}(\lambda, \beta, \alpha)[21]$.

(3) $0-\mathscr{V} \mathscr{U}_{0}(\alpha, 1,0,1)=\mathscr{C} \mathscr{V}(\alpha)[11]$.

(4) $k-\mathscr{V} \mathscr{U}_{0}(\alpha, 1,0,1)=k-\mathscr{U} \mathscr{C V}(\alpha)[23]$.

(5) $1-\mathscr{U}(\alpha, 1,0,1)=\mathscr{U} \mathscr{C} \mathscr{V}(\alpha)[4]$.

(6) $k-\mathscr{U}(\alpha, \beta, 0,0)=k-\mathscr{U} \mathcal{S} \mathscr{T}(\alpha, \beta)[13]$.

Throughout the paper $-1 \leq \alpha \leq \beta \leq 1,0 \leq \lambda<1$, $1-\alpha>k(1-\beta)$, and $z \in \Delta$, unless otherwise stated.

\section{Main Results}

Theorem 2. A function $f(z)$ given by (1) is in class $k$ $\mathcal{U}(\alpha, \beta, \lambda, \delta)$ if

$$
\begin{aligned}
\sum_{n=2}^{\infty} & {\left[\Pi_{n}(1+k)-(k \beta+\alpha) \Omega_{n}\right]\left|a_{n}\right| } \\
& \leq 1-\alpha-k(1-\beta),
\end{aligned}
$$

where

$$
\begin{aligned}
& \Pi_{n}=(n+\delta n(n-1)(1+\lambda n)), \\
& \Omega_{n}=1-\delta+\delta n(1+\lambda(n-1)) .
\end{aligned}
$$

Proof. It is sufficient to prove that inequality (9) holds. As we know

$$
\begin{aligned}
& \operatorname{Re}(w)>k|w-\beta|+\alpha \\
& \quad \text { iff } \operatorname{Re}\left(\left(1+k e^{i \theta}\right) w-\beta k e^{i \theta}\right) \geq \alpha ;
\end{aligned}
$$


then inequality (9) can be written as

$$
\begin{aligned}
& \operatorname{Re}\left(\left(1+k e^{i \theta}\right)\right. \\
& \cdot \frac{(1-\delta) z f^{\prime}+\delta\left(z f^{\prime}+(1+2 \lambda) z^{2} f^{\prime \prime}+\lambda z^{3} f^{\prime \prime \prime}\right)}{(1-\delta) f+\delta\left(z f^{\prime}+\lambda z^{2} f^{\prime \prime}\right)} \\
& \left.-\beta k e^{i \theta}\right) \geq \alpha .
\end{aligned}
$$

This is,

$$
\operatorname{Re}\left(\frac{A(z)}{B(z)}\right) \geq \alpha
$$

where

$$
\begin{aligned}
A(z) & \\
= & \left(1+k e^{i \theta}\right)\left(z f^{\prime}+\delta\left((1+2 \lambda) z^{2} f^{\prime \prime}+\lambda z^{3} f^{\prime \prime \prime}\right)\right) \\
& -\beta k e^{i \theta}\left((1-\delta) f+\delta\left(z f^{\prime}+\lambda z^{2} f^{\prime \prime}\right)\right), \\
B(z)= & (1-\delta) f+\delta\left(z f^{\prime}+\lambda z^{2} f^{\prime \prime}\right) ;
\end{aligned}
$$

then we have

$$
|A(z)+(1-\alpha) B(z)|-|A(z)-(1+\alpha) B(z)| \geq 0 .
$$

Now

$$
\begin{aligned}
& |A(z)+(1-\alpha) B(z)|=\mid(1-\beta) k e^{i \theta}+(2-\alpha) z \\
& \quad-\sum_{n=2}^{\infty}\left[\left(\beta \Omega_{n}-\Pi_{n}\right) k e^{i \theta}-(1-\alpha) \Omega_{n}-\Pi_{n}\right] a_{n} z^{n} \mid \\
& \quad \geq-((1-\beta) k+(2-\alpha))|z| \\
& \quad-\sum_{n=2}^{\infty}\left[\left(\beta \Omega_{n}-\Pi_{n}\right) k+(1-\alpha) \Omega_{n}+\Pi_{n}\right]\left|a_{n}\right||z|^{n},
\end{aligned}
$$

and also

$$
\begin{aligned}
& |A(z)-(1+\alpha) B(z)|=\mid\left((1-\beta) k e^{i \theta}-\alpha\right) z \\
& \quad+\sum_{n=2}^{\infty}\left[\left(\Pi_{n}-\beta \Omega_{n}\right) k e^{i \theta}-(1+\alpha) \Omega_{n}+\Pi_{n}\right] a_{n} z^{n} \mid \\
& \quad \leq((1-\beta) k+\alpha)|z| \\
& \quad+\sum_{n=2}^{\infty}\left[\left(\Pi_{n}-\beta \Omega_{n}\right) k-(1+\alpha) \Omega_{n}+\Pi_{n}\right]\left|a_{n}\right|\left|z^{n}\right| .
\end{aligned}
$$

From (18) and (19), we have

$$
\begin{aligned}
& |A(z)+(1-\alpha) B(z)|-|A(z)-(1+\alpha) B(z)| \\
& \quad \geq 2[(1-\alpha)-k(1-\beta)]|z| \\
& \quad-2 \sum_{n=2}^{\infty}\left[\left(\Pi_{n}-\beta \Omega_{n}\right) k+\left(\Pi_{n}-\alpha \Omega_{n}\right)\right]\left|a_{n}\right||z|^{n} \\
& \quad=2[[(1-\alpha)-k(1-\beta)]|z| \\
& \left.\quad-\sum_{n=2}^{\infty}\left[\Pi_{n}(1+k)-(k \beta+\alpha) \Omega_{n}\right]\left|a_{n}\right||z|^{n}\right] .
\end{aligned}
$$

The last expression is bounded below by 0 if

$$
\begin{aligned}
& \sum_{n=2}^{\infty}\left[\Pi_{n}(1+k)-(k \beta+\alpha) \Omega_{n}\right]\left|a_{n}\right| \\
& \quad \leq 1-\alpha-k(1-\beta),
\end{aligned}
$$

which completes the proof.

In the next theorem, we prove that condition (11) is also necessary for function $f \in k-\mathcal{U}(\alpha, \beta, \lambda, \delta)$.

Theorem 3. Let $f(z)$ be given by (1) and in $V_{\eta}$; then $f \in k-$ $\mathscr{V} \mathscr{U}_{\eta}(\alpha, \beta, \lambda, \delta)$ if and only if

$$
\begin{aligned}
& \sum_{n=2}^{\infty}\left[\Pi_{n}(1+k)-(k \beta+\alpha) \Omega_{n}\right]\left|a_{n}\right| \\
& \quad \leq 1-\alpha-k(1-\beta) .
\end{aligned}
$$

Proof. From Theorem 2, we need only to show that $f \in$ $k-\mathscr{V} \mathscr{U}_{\eta}(\alpha, \beta, \lambda, \delta)$ satisfies inequality (22). If $f \in k-$ $\mathscr{V} \mathscr{U}_{\eta}(\alpha, \beta, \lambda, \delta)$, then by definition, we have

$$
\begin{aligned}
& \operatorname{Re}\left(\frac{(1-\alpha)+\sum_{n=2}^{\infty}\left(\Pi_{n}-\alpha \Omega_{n}\right) a_{n} z^{n-1}}{1+\sum_{n=2}^{\infty} \Omega_{n} a_{n} z^{n-1}}\right) \\
& \quad \geq k\left|\frac{(1-\beta)+\sum_{n=2}^{\infty}\left(\Pi_{n}-\beta \Omega_{n}\right) a_{n} z^{n-1}}{1+\sum_{n=2}^{\infty} \Omega_{n} a_{n} z^{n-1}}\right| .
\end{aligned}
$$

Since $f$ is function of form (1) with the argument property given in class $V_{\eta}$ and letting $z=r e^{\theta}$ in the above inequality, we have

$$
\begin{aligned}
& \frac{(1-\alpha)-\sum_{n=2}^{\infty}\left(\Pi_{n}-\alpha \Omega_{n}\right)\left|a_{n}\right| r^{n-1}}{1-\sum_{n=2}^{\infty} \Omega_{n}\left|a_{n}\right| r^{n-1}} \\
& \geq k\left(\frac{(1-\beta)-\sum_{n=2}^{\infty}\left(\Pi_{n}-\beta \Omega_{n}\right)\left|a_{n}\right| r^{n-1}}{1-\sum_{n=2}^{\infty} \Omega_{n}\left|a_{n}\right| r^{n-1}}\right)
\end{aligned}
$$

for $r \rightarrow 1$, and (24) leads to require inequality

$$
\begin{aligned}
& \sum_{n=2}^{\infty}\left[\Pi_{n}(1+k)-(k \beta+\alpha) \Omega_{n}\right]\left|a_{n}\right| \\
& \quad \leq 1-\alpha-k(1-\beta) .
\end{aligned}
$$


The function

$$
\begin{gathered}
f_{n, \eta}(z)=z-\frac{(1-\alpha-k(1-\beta)) e^{i(1-n) \eta}}{\Pi_{n}(1+k)-(k \beta+\alpha) \Omega_{n}} z^{n}, \\
0 \leq \eta \leq 2 \pi, n \geq 2
\end{gathered}
$$

is extremal function.

Corollary 4. Let $f(z)$ given in (1) be in class $k-\mathscr{V} \mathscr{U}_{\eta}(\alpha, \beta$, $\lambda, \delta)$. Then

$$
\left|a_{n}\right| \leq \frac{1-\alpha-k(1-\beta)}{\Pi_{n}(1+k)-(k \beta+\alpha) \Omega_{n}}, \quad n \geq 2 .
$$

Inequality (27) is attained for the function given in (26).

Theorem 5. Let the function $f(z)$ given in (1) be in class $k-$ $\mathscr{V} \mathcal{U}_{\eta}(\alpha, \beta, \lambda, \delta)$. Then for $|z|<r=1$

$$
\begin{aligned}
& |f(z)| \geq r-\frac{1-\alpha-k(1-\beta)}{\Pi_{2}(1+k)-(k \beta+\alpha) \Omega_{2}} r^{2}, \\
& |f(z)| \leq r+\frac{1-\alpha-k(1-\beta)}{\Pi_{2}(1+k)-(k \beta+\alpha) \Omega_{2}} r^{2} .
\end{aligned}
$$

The results in (28) are attained for the function given in (26) for $z= \pm r$.

Proof. As we know from Theorem 3

$$
\begin{aligned}
& {\left[\Pi_{2}(1+k)-(k \beta+\alpha) \Omega_{2}\right] \sum_{n=2}^{\infty}\left|a_{n}\right|} \\
& \quad \leq \sum_{n=2}^{\infty}\left[\Pi_{n}(1+k)-(k \beta+\alpha) \Omega_{n}\right]\left|a_{n}\right| \\
& \quad \leq 1-\alpha-k(1-\beta) .
\end{aligned}
$$

As

$$
\begin{aligned}
|f(z)| & \geq|z|-\sum_{n=2}^{\infty}\left|a_{n}\right||z|^{n} \geq r-r^{2} \sum_{n=2}^{\infty}\left|a_{n}\right| \\
& \geq r-\frac{1-\alpha-k(1-\beta)}{\Pi_{2}(1+k)-(k \beta+\alpha) \Omega_{2}} r^{2},
\end{aligned}
$$

similarly

$$
\begin{aligned}
|f(z)| & \leq|z|+\sum_{n=2}^{\infty}\left|a_{n}\right||z|^{n} \leq r+r^{2} \sum_{n=2}^{\infty}\left|a_{n}\right| \\
& \leq r+\frac{1-\alpha-k(1-\beta)}{\Pi_{2}(1+k)-(k \beta+\alpha) \Omega_{2}} r^{2} .
\end{aligned}
$$

This completes the proof.
Theorem 6. Let the function $f(z)$ given in (1) be in class $k-$ $\mathscr{V} \mathcal{U}_{\eta}(\alpha, \beta, \lambda, \delta)$. Then for $|z|<r=1$

$$
\begin{gathered}
1-\frac{2(1-\alpha-k(1-\beta))}{\Pi_{2}(1+k)-(k \beta+\alpha) \Omega_{2}} r \leq|f(z)| \\
\leq 1+\frac{2(1-\alpha-k(1-\beta))}{\Pi_{2}(1+k)-(k \beta+\alpha) \Omega_{2}} r .
\end{gathered}
$$

Proof. For $f(z)$ given by (1), we have

$$
\begin{aligned}
& \left|f^{\prime}(z)\right| \geq 1-\sum_{n=2}^{\infty} n\left|a_{n}\right||z|^{n-1} \geq 1-r \sum_{n=2}^{\infty} n\left|a_{n}\right|, \\
& \left|f^{\prime}(z)\right| \leq 1+\sum_{n=2}^{\infty} n\left|a_{n}\right||z|^{n-1} \leq 1+r \sum_{n=2}^{\infty} n\left|a_{n}\right| .
\end{aligned}
$$

In view of Theorem 3 ,

$$
\begin{aligned}
& \frac{\left[\Pi_{2}(1+k)-(k \beta+\alpha) \Omega_{2}\right]}{2} \sum_{n=2}^{\infty} n\left|a_{n}\right| \\
& \quad \leq \sum_{n=2}^{\infty}\left[\Pi_{n}(1+k)-(k \beta+\alpha) \Omega_{n}\right]\left|a_{n}\right| \\
& \quad \leq 1-\alpha-k(1-\beta),
\end{aligned}
$$

or, equivalently,

$$
\sum_{n=2}^{\infty} n\left|a_{n}\right| \leq \frac{2(1-\alpha-k(1-\beta))}{\left[\Pi_{2}(1+k)-(k \beta+\alpha) \Omega_{2}\right]} .
$$

A substitution from (35) into (33) yields inequality (32), which is required.

Theorem 7. Let $f \in k-\mathscr{V} \mathcal{U}_{\eta}(\alpha, \beta, \lambda, \delta)$ with argument property as in class $V_{\eta}$. Define $f_{j}(z)=z$ and

$$
f_{n, \eta}=z-\frac{1-\alpha-k(1-\beta) e^{i(1-n) \eta}}{\left[\Pi_{n}(1+k)-(k \beta+\alpha) \Omega_{n}\right]} z^{n},
$$

where $0 \leq \eta \leq 2 \pi, n \geq 2$.

Then function $f(z)$ is in class $k-\mathscr{V} \mathscr{U}_{\eta}(\alpha, \beta, \lambda, \delta)$ if and only if it can be expressed as

$$
f(z)=\sum_{n=1}^{\infty} \mu_{n} f_{n, \eta},
$$

where $\mu_{n} \geq 0(n \geq 1)$ and $\sum_{n=1}^{\infty} \mu_{n}=1$.

Proof. Assume that

$$
f(z)=\mu_{1} f_{1}(z)
$$

$$
\begin{aligned}
& +\sum_{n=2}^{\infty} \mu_{n}\left[z-\frac{1-\alpha-k(1-\beta) e^{i(1-n) \eta}}{\Pi_{n}(1+k)-(k \beta+\alpha) \Omega_{n}} z^{n}\right] \\
= & \sum_{n=1}^{\infty} \mu_{n} z
\end{aligned}
$$


Then it follows that

$$
\begin{aligned}
\sum_{n=2}^{\infty}\left|\frac{1-\alpha-k(1-\beta) e^{i(1-n) \eta}}{\Pi_{n}(1+k)-(k \beta+\alpha) \Omega_{n}}\right| & \cdot \mu_{n}\left[\Pi_{n}(1+k)-(k \beta+\alpha) \Omega_{n}\right] \\
= & \sum_{n=2}^{\infty} \mu_{n}[1-\alpha-k(1-\beta)] \leq\left(1-\mu_{1}\right) \\
\cdot & {[1-\alpha-k(1-\beta)] \leq 1-\alpha-k(1-\beta), }
\end{aligned}
$$

by Theorem 3, $f \in k-\mathscr{V} \mathcal{U}_{\eta}(\alpha, \beta, \lambda, \delta)$. Conversely, assume that the function $f(z)$ defined by (1) belongs to class $k-$ $\mathscr{V} \mathscr{U}_{\eta}(\alpha, \beta, \lambda, \delta)$, and then

$$
\left|a_{n}\right| \leq \frac{1-\alpha-k(1-\beta)}{\Pi_{n}(1+k)-(k \beta+\alpha) \Omega_{n}}, \quad n \geq 2 .
$$

Set

$$
\mu_{n}=\frac{\Pi_{n}(1+k)-(k \beta+\alpha) \Omega_{n}}{1-\alpha-k(1-\beta)}\left|a_{n}\right|, \quad n \geq 2,
$$

and $\mu_{1}=1-\sum_{n=2}^{\infty} \mu_{n}, n \geq 2$. Then $f(z)=\sum_{n=1}^{\infty} \mu_{n} f_{n, \eta}$ and this completes the proof.

Theorem 8. Let $f \in k-\mathscr{V} \mathscr{U}_{\eta}(\alpha, \beta, \lambda, \delta)$. Then $f(z)$ is close to convex of order $\sigma(0 \leq \sigma<1)$ in the disc $|z|<r_{1}$, where

$$
r_{1}=\inf \left[\frac{(1-\sigma)\left(\Pi_{n}(1+k)-(k \beta+\alpha) \Omega_{n}\right)}{n(1-\alpha-k(1-\beta))}\right]^{1 /(n-1)},
$$

$$
n \geq 2 \text {. }
$$

Proof. As $f \in V_{\eta}$, where $f$ is close to convex of order $\sigma$, we have

$$
\left|f^{\prime}(z)-1\right|<1-\sigma
$$

as

$$
\left|f^{\prime}(z)-1\right| \leq \sum_{n=2}^{\infty} n\left|a_{n}\right||z|^{n-1}
$$

this expression is less than $1-\sigma$ if

$$
\sum_{n=2}^{\infty} \frac{n}{1-\sigma}\left|a_{n}\right||z|^{n-1}<1
$$

By the fact that $f \in k-\mathscr{V} \mathcal{U}_{\eta}(\alpha, \beta, \lambda, \delta)$ if and only if

$$
\sum_{n=2}^{\infty}\left[\frac{\Pi_{n}(1+k)-(k \beta+\alpha) \Omega_{n}}{(1-\alpha-k(1-\beta))}\right] a_{n} \leq 1
$$

inequality (43) is true if

$$
\frac{n}{1-\sigma} z^{n-1} \leq \frac{\Pi_{n}(1+k)-(k \beta+\alpha) \Omega_{n}}{(1-\alpha-k(1-\beta))}
$$

or, equivalently,

$$
|z|^{n-1}=\left[\frac{(1-\sigma)\left(\Pi_{n}(1+k)-(k \beta+\alpha) \Omega_{n}\right)}{n(1-\alpha-k(1-\beta))}\right] .
$$

Theorem 9. Let $f \in k-\mathscr{V} \mathcal{U}_{\eta}(\alpha, \beta, \lambda, \delta)$. Then $f(z)$ is close to convex of order $\sigma(0 \leq \sigma<1)$ in the $\operatorname{disc}|z|<r_{2}$, where

$$
r_{2}=\inf \left[\frac{(1-\sigma)\left(\Pi_{n}(1+k)-(k \beta+\alpha) \Omega_{n}\right)}{(n-\sigma)(1-\alpha-k(1-\beta))}\right]^{1 /(n-1)},
$$

$n \geq 2$

Proof. As $f \in V_{\eta}$ and $f$ is starlike of order $\sigma$, then we have

$$
\left|\frac{z f^{\prime}(z)}{f(z)}-1\right|<1-\sigma
$$

as

$$
\left|\frac{z f^{\prime}(z)}{f(z)}-1\right| \leq \frac{\sum_{n=2}^{\infty}(n-1)\left|a_{n}\right||z|^{n-1}}{1-\sum_{n=2}^{\infty}\left|a_{n}\right||z|^{n-1}} .
$$

The last expression is less than $1-\sigma$ if

$$
\sum_{n=2}^{\infty} \frac{n-\sigma}{1-\sigma}\left|a_{n}\right||z|^{n-1}<1 .
$$

Using the fact that $f \in k-\mathscr{V} \mathcal{U}_{\eta}(\alpha, \beta, \lambda, \delta)$ if and only if

$$
\sum_{n=2}^{\infty}\left[\frac{\Pi_{n}(1+k)-(k \beta+\alpha) \Omega_{n}}{(1-\alpha-k(1-\beta))}\right] a_{n} \leq 1
$$

(50) is true if

$$
\frac{n-\sigma}{1-\sigma}|z|^{n-1}<\frac{\Pi_{n}(1+k)-(k \beta+\alpha) \Omega_{n}}{(1-\alpha-k(1-\beta))} .
$$

Or equivalently

$$
|z|^{n-1}=\frac{(1-\sigma)\left(\Pi_{n}(1+k)-(k \beta+\alpha) \Omega_{n}\right)}{(n-\sigma)(1-\alpha-k(1-\beta))},
$$

which is required.

Theorem 10. Let $f \in k-\mathscr{V} \mathcal{U}_{\eta}(\alpha, \beta, \lambda, \delta)$. Then $f(z)$ is convex of order $\sigma(0 \leq \sigma<1)$ in the disc $|z|<r_{3}$, where

$$
r_{3}=\inf \left[\frac{(1-\sigma)\left[\Pi_{n}(1+k)-(k \beta+\alpha) \Omega_{n}\right]}{n(n-\sigma)(1-\alpha-k(1-\beta))}\right]^{1 /(n-1)},
$$

$n \geq 2$.

Proof. Using the fact that $f$ is convex if and only if $z f^{\prime}$ is starlike, following the lines of Theorem 9, we have the required results.

Theorem 11. Let $f_{j}(z)(j=1,2, \ldots)$ given by (6) be in class $k-\mathscr{V} \mathcal{U}_{\eta}(\alpha, \beta, \lambda, \delta)$. Then $\left(f_{1} * f_{2}\right) \in k-\mathscr{V} \mathscr{U}_{\eta}\left(\phi_{1}, \lambda, \delta\right)$, for 


$$
\phi_{1}=\frac{\left(\Pi_{n}(1+k)-(k \beta+\alpha) \Omega_{n}\right)^{2}-\left(\Pi_{n}(1+k)-k \beta \Omega_{n}\right)(1-\alpha-k(1-\beta))^{2}}{\left(\Pi_{n}(1+k)-(k \beta+\alpha) \Omega_{n}\right)^{2}-\Omega_{n}(1-\alpha-k(1-\beta))^{2}} .
$$

Proof. We need to prove the largest $\phi_{1}$ such that

$$
\frac{\left(\Pi_{n}(1+k)-(k \beta+\alpha) \Omega_{n}\right)}{\left(1-\phi_{1}-k(1-\beta)\right)} a_{n, 1} a_{n, 2} \leq 1 .
$$

From Theorem 3, we have

$$
\begin{aligned}
& \sum_{n=2}^{\infty}\left[\frac{\Pi_{n}(1+k)-(k \beta+\alpha) \Omega_{n}}{(1-\alpha-k(1-\beta))}\right] a_{n, 1} \leq 1, \\
& \sum_{n=2}^{\infty}\left[\frac{\Pi_{n}(1+k)-(k \beta+\alpha) \Omega_{n}}{(1-\alpha-k(1-\beta))}\right] a_{n, 2} \leq 1 .
\end{aligned}
$$

By Cauchy-Schwarz inequality, we have

$$
\sum_{n=2}^{\infty}\left[\frac{\Pi_{n}(1+k)-(k \beta+\alpha) \Omega_{n}}{(1-\alpha-k(1-\beta))}\right] \sqrt{a_{n, 1} a_{n, 2}} \leq 1
$$

Thus, it is sufficient to show

$$
\begin{aligned}
& {\left[\frac{\Pi_{n}(1+k)-\left(k \beta+\phi_{1}\right) \Omega_{n}}{\left(1-\phi_{1}-k(1-\beta)\right)}\right] a_{n, 1} a_{n, 2}} \\
& \quad \leq\left[\frac{\Pi_{n}(1+k)-(k \beta+\alpha) \Omega_{n}}{(1-\alpha-k(1-\beta))}\right] \sqrt{a_{n, 1} a_{n, 2}}, \quad n \geq 2 .
\end{aligned}
$$

For $n \geq 2$

$$
\begin{aligned}
& \sqrt{a_{n, 1} a_{n, 2}} \\
& \quad \leq \frac{\left(\Pi_{n}(1+k)-(k \beta+\alpha) \Omega_{n}\right)\left(1-\phi_{1}-k(1-\beta)\right)}{\left(\Pi_{n}(1+k)-\left(k \beta+\phi_{1}\right) \Omega_{n}\right)(1-\alpha-k(1-\beta))} .
\end{aligned}
$$

Note that

$$
\sqrt{a_{n, 1} a_{n, 2}} \leq \frac{(1-\alpha-k(1-\beta))}{\left(\Pi_{n}(1+k)-(k \beta+\alpha) \Omega_{n}\right)}
$$

We need to show

$$
\begin{aligned}
& \frac{(1-\alpha-k(1-\beta))}{\left(\Pi_{n}(1+k)-(k \beta+\alpha) \Omega_{n}\right)} \\
& \quad \leq \frac{\left(\Pi_{n}(1+k)-(k \beta+\alpha) \Omega_{n}\right)\left(1-\phi_{1}-k(1-\beta)\right)}{\left(\Pi_{n}(1+k)-\left(k \beta+\phi_{1}\right) \Omega_{n}\right)(1-\alpha-k(1-\beta))},
\end{aligned}
$$

or equivalently

$$
\phi_{1} \leq \frac{\left(\Pi_{n}(1+k)-(k \beta+\alpha) \Omega_{n}\right)^{2}-\left(\Pi_{n}(1+k)-k \beta \Omega_{n}\right)(1-\alpha-k(1-\beta))^{2}}{\left(\Pi_{n}(1+k)-(k \beta+\alpha) \Omega_{n}\right)^{2}-\Omega_{n}(1-\alpha-k(1-\beta))^{2}}=\omega(n) .
$$

$\omega(n)$ is an increasing function for $n \geq 2$. For $n=2$ in (65),

$$
\phi_{1} \leq \omega(2)=\frac{\left(\Pi_{2}(1+k)-(k \beta+\alpha) \Omega_{2}\right)^{2}-\left(\Pi_{2}(1+k)-k \beta \Omega_{2}\right)(1-\alpha-k(1-\beta))^{2}}{\left(\Pi_{2}(1+k)-(k \beta+\alpha) \Omega_{2}\right)^{2}-\Omega_{2}(1-\alpha-k(1-\beta))^{2}}
$$

which proves main assertion of Theorem 11.

\section{Conflicts of Interest}

The authors declare that they have no conflicts of interest.

\section{Authors' Contributions}

All authors jointly work on the results, and they read and approved the final manuscript.

\section{Acknowledgments}

The work here is supported by MOHE Grant: FRGS/1/2016/ STG06/UKM/01/1.

\section{References}

[1] A. W. Goodman, "On uniformly convex functions," Annales Polonici Mathematici, vol. 56, no. 1, pp. 87-92, 1991.

[2] A. W. Goodman, "On uniformly starlike functions," Journal of Mathematical Analysis and Applications, vol. 155, no. 2, pp. 364-370, 1991. 
[3] W. C. Ma and D. Minda, "Uniformly convex functions," Annales Polonici Mathematici, vol. 57, no. 2, pp. 165-175, 1992.

[4] F. Rønning, "Uniformly convex functions and a corresponding class of starlike functions," Proceedings of the American Mathematical Society, vol. 118, no. 1, pp. 189-196, 1993.

[5] J. Sokol and A. Wisniowska-Wajnryb, "On some classes of starlike functions related with parabola," Folia Sci. Univ. Tech. Resov., vol. 121, no. 18, pp. 35-42, 1993.

[6] J. Sokol and A. Wisniowska-Wajnryb, "On certain problem in the classes of $k$-starlike functions," Computers \& Mathematics with Applications, vol. 62, no. 12, pp. 4733-4741, 2011.

[7] S. Kanas and A. Wisniowska, "Conic regions and $k$-uniform convexity," Journal of Computational and Applied Mathematics, vol. 105, no. 1-2, pp. 327-336, 1999.

[8] S. Kanas and H. M. Srivastava, "Linear operators associated with k-uniformly convex functions," Integral Transforms and Special Function, vol. 9, no. 2, pp. 121-132, 2000.

[9] A. Mannino, "Some inequalities concerning starlike and convex functions," General Mathematics, vol. 12, no. 1, pp. 5-12, 2004.

[10] S. Ponnusamy and M. Vuorinen, "Univalence and convexity properties for Gaussian hypergeometric functions," The Rocky Mountain Journal of Mathematics, vol. 31, no. 1, pp. 327-353, 2001.

[11] H. Silverman, "Univalent functions with negative coefficients," Proceedings of the American Mathematical Society, vol. 51, pp. 109-116, 1975.

[12] F. Ronning, "Integral representation for bounded starlike functions," Annales Polonici Mathematici, vol. 60, no. 3, pp. 289-297, 1995.

[13] R. M. El-Ashwah, M. K. Aouf, A. A. Hassan, and A. H. Hassan, "Certain new classes of analytic functions with varying arguments," Journal of Complex Analysis, vol. 2013, Article ID 958210, 5 pages, 2013.

[14] R. M. Ali, S. R. Mondal, and V. Ravichandran, "On the Janowski convexity and starlikeness of the confluent hypergeometric function," Bulletin of the Belgian Mathematical Society. Simon Stevin, vol. 22, no. 2, pp. 227-250, 2015.

[15] R. M. Ali, V. Ravichandran, and N. Seenivasagan, "Subordination and superordination of the Liu-Srivastava linear operator on meromorphic functions," Bulletin of the Malaysian Mathematical Sciences Society, vol. 31, no. 2, pp. 193-207, 2008.

[16] R. M. Ali and V. Ravichandran, "Uniformly convex and uniformly starlike functions," Mathematics Newsletter, vol. 21, pp. 16-30, 2011.

[17] Ş. Altınkaya and S. Yalçın, "Coefficient estimates for two new subclasses of bi-univalent functions with respect to symmetric points," Journal of Function Spaces, Article ID 145242, 2014.

[18] M. K. Aouf, H. M. Hossen, and A. Y. Lashin, "On certain families of analytic functions with negative coefficients," Indian Journal of Pure and Applied Mathematics, vol. 31, no. 8, pp. 9991015, 2000.

[19] M. K. Aouf, A. A. Shamandy, A. O. Mostafa, and A. K. Wagdy, "Certain subclasses of uniformly starlike and convex functions defined by convolution with negative coefficients," Matematichki Vesnik, vol. 65, no. 1, pp. 14-28, 2013.

[20] A. Kamiński and S. Mincheva-Kaminska, "Compatibility conditions and the convolution of functions and generalized functions," Journal of Function Spaces and Applications, vol. 2013, Article ID 356724, 11 pages, 2013.

[21] N. Magesh, "Certain subclasses of uniformly convex functions of order $\alpha$ and type $\beta$ with varying arguments," Journal of the Egyptian Mathematical Society, vol. 21, no. 3, pp. 184-189, 2013.
[22] K. I. Noor, "Some properties of certain analytic functions," Journal of Natural Geometry, vol. 7, no. 1, pp. 11-20, 1995.

[23] R. Bharati, R. Parvatham, and A. Swaminathan, "On subclasses of uniformly convex functions and corresponding class of starlike functions," Tamkang Journal of Mathematics, vol. 28, no. 1, pp. 17-32, 1997. 


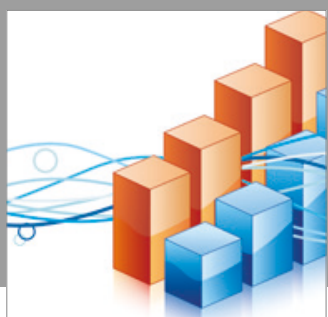

Advances in

Operations Research

vatersals

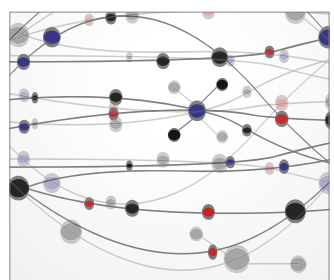

\section{The Scientific} World Journal
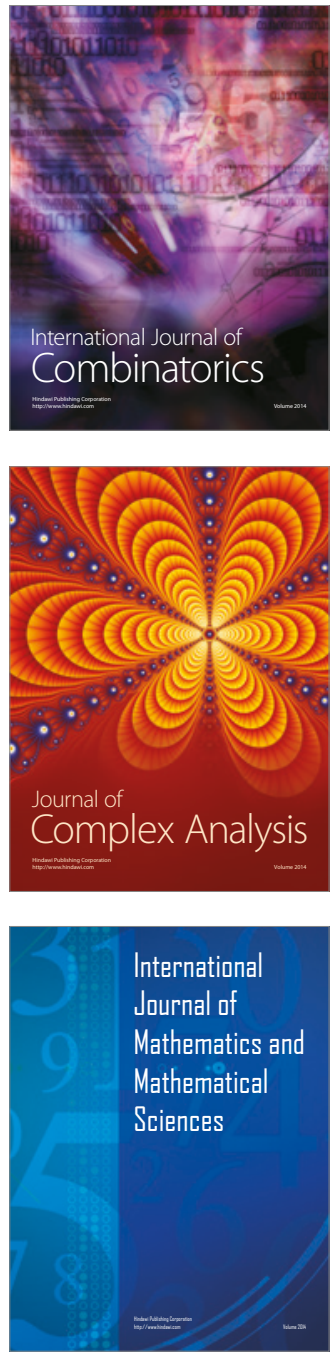
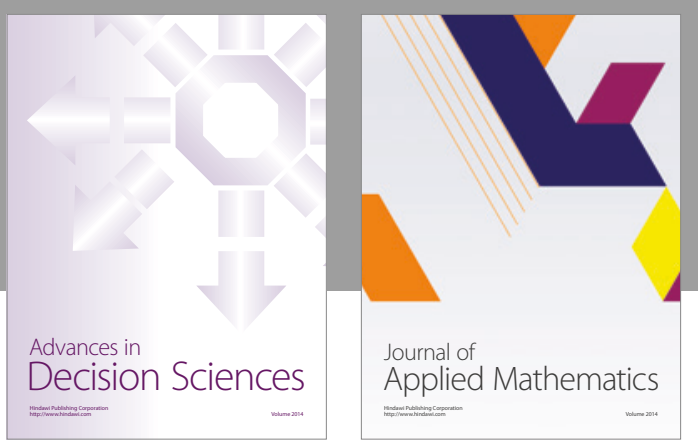

Algebra

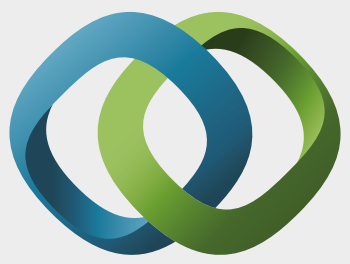

\section{Hindawi}

Submit your manuscripts at

https://www.hindawi.com
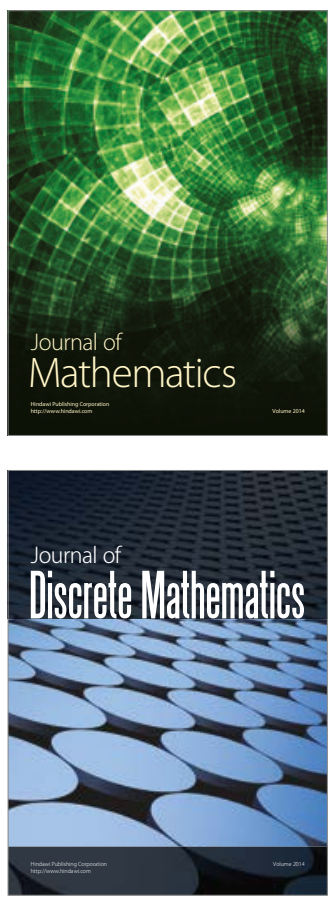

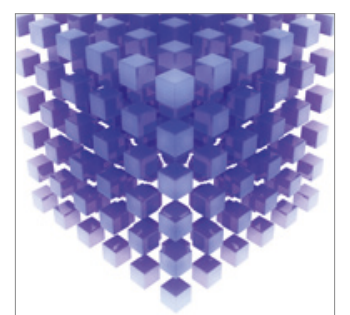

Mathematical Problems in Engineering
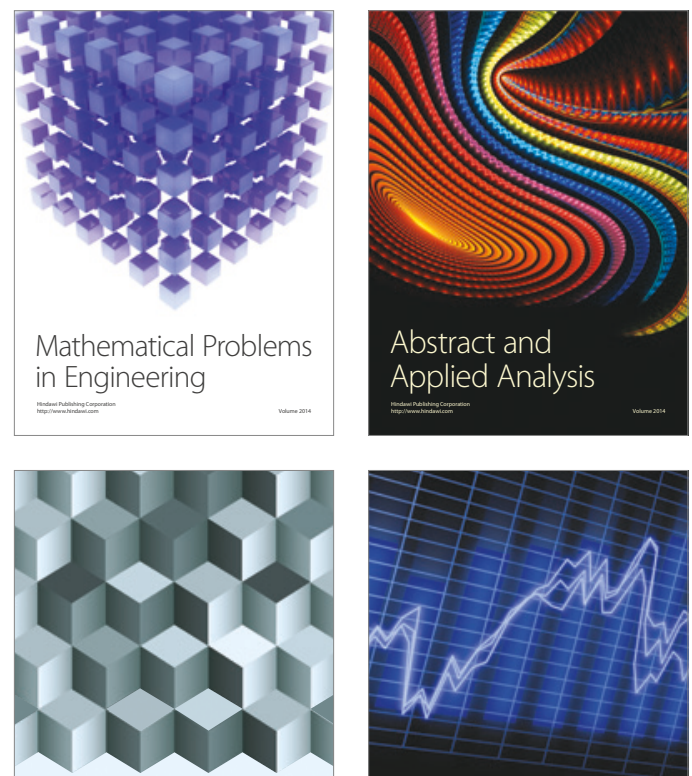

Journal of

Function Spaces

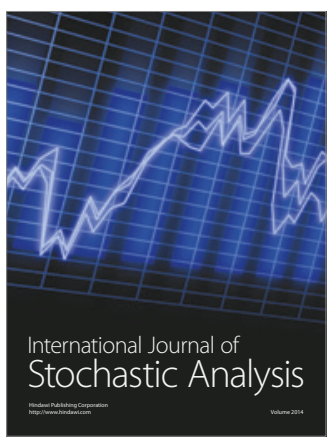

Probability and Statistics
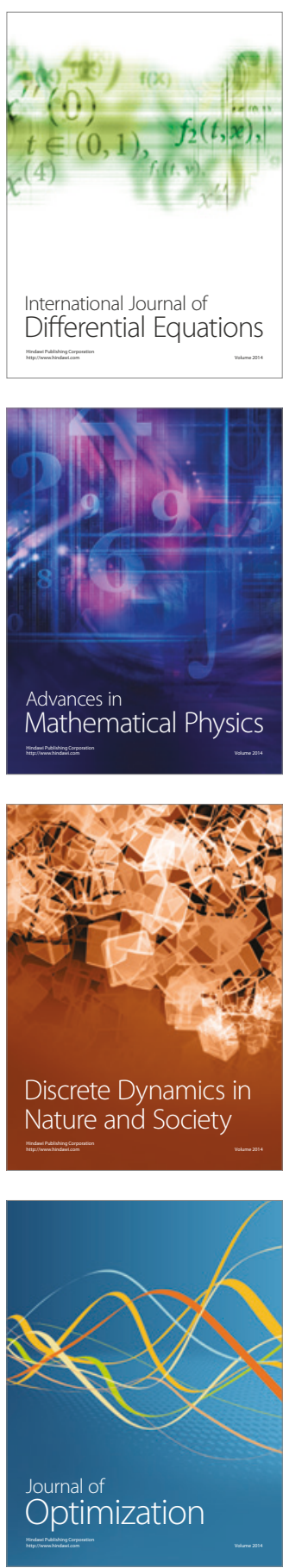